\title{
Incontinência urinária e noctúria: prevalência e impacto sobre qualidade de vida em idosas numa Unidade Básica de Saúde
}

\author{
Urinary incontinence and nocturia: prevalence and impact on quality of life in elderly women in a \\ Primary Health Care Unit
}

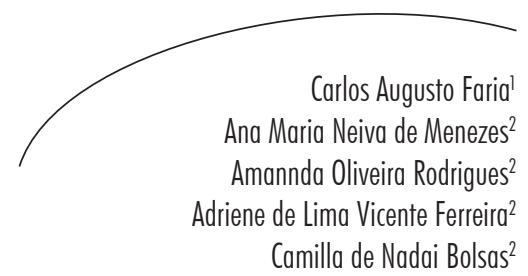

Resumo

Objetivos: estimar a prevalência de incontinência urinária e de seus subtipos (incontinência urinária de esforço, bexiga hiperativa e incontinência mista), a prevalência do sintoma de noctúria, e avaliar o impacto dessas condições sobre a qualidade de vida na população de idosas atendida para vacinação numa Unidade Básica de Saúde de Niterói-RJ. Métodos: estudo observacional descritivo, com utilização das versões brasileiras do International Consultation on Incontinence Questionnaire - Short Form e do King's Health Questionnaire, respectivamente, para triagem de mulheres com incontinência urinária e para avaliar o impacto da incontinência urinária e da noctúria sobre a qualidade de vida. Participaram do estudo 66 mulheres. Resultados: a média das

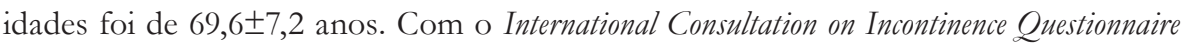
- Short Form, a prevalência de incontinência urinária foi de $42,4 \%$, sendo que $42,9 \%$ das idosas incontinentes referiram que a perda interferia nas suas atividades diárias. A prevalência de incontinência urinária de esforço, bexiga hiperativa e incontinência mista foi de $15,2 \%, 12,1 \%$ e 10,6\%, respectivamente. Dentre as mulheres incontinentes, 20 aceitaram responder ao King's Health Questionnaire, tinham incontinência mista 11 delas $(55 \%)$ e 16 apresentavam noctúria $(80 \%)$. Houve comprometimento da qualidade de vida em todos os domínios. Conclusão: a prevalência de incontinência urinária foi elevada na população estudada. Com a utilização do questionário de triagem, a incontinência urinária de esforço foi o subtipo mais comum, ao passo que a utilização do questionário de avaliação de qualidade de vida mostrou prevalência mais elevada de incontinência mista. A frequência de noctúria foi estimada somente para as mulheres que responderam ao King's Health Questionnaire. Houve comprometimento da qualidade de vida em todos os domínios avaliados.

\footnotetext{
Departamento Materno-infantil, Faculdade de Medicina. Universidade Federal Fluminense. Niterói, RJ, Brasil.

2 Programa de Iniciação Científica, Faculdade de Medicina. Universidade Federal Fluminense. Niterói, RJ, Brasil.
}

\author{
Palavras-chave: \\ Incontinência Urinária. \\ Incontinência Urinária por \\ Estresse. Noctúria. Qualidade \\ de Vida. Saúde do Idoso.
}




\section{Abstract}

Objectives: To evaluate the prevalence of urinary incontinence and its subtypes stress urinary incontinence, overactive bladder and mixed urinary incontinence, the prevalence of nocturia, and the impact of these conditions on quality of life in an elderly female population attended for vaccination in a Primary Health Care Unit in Niterói city, State of Rio de Janeiro, Brazil. Methods: In an observational descriptive study, the Brazilian versions of the International Consultation on Incontinence Questionnaire - Short Form and of the King's Health Questionnaire were used respectively to do the screening of women with urinary incontinence and to evaluate the impact of urinary incontinence and nocturia on quality of life. Sixty-six women took part in the survey. Results: The average age was $69.6 \pm 7.2$ years. According to the International Consultation on Incontinence Questionnaire - Short Form, the prevalence of IU was $42.4 \%$, and $42.9 \%$ of elderly incontinent women reported impact of incontinence on their daily activities. The prevalence of stress urinary incontinence, overactive bladder and mixed urinary incontinence was, respectively, 15.2\%,12.1\% and 10.6\%. Twenty incontinent women agreed to answer the King's Health Questionnaire. Eleven of them $(55 \%)$ reported complaints of mixed incontinence, and sixteen women $(80 \%)$ presented nocturia. It was observed impact in quality of life in all domains. Conclusion: It was observed high prevalence of urinary incontinence in the studied population. According to the screening questionnaire, the most prevalent subtype of incontinence was stress urinary incontinence, whilst the use of the quality of life questionnaire showed higher prevalence of mixed incontinence. The frequency of nocturia could be estimated only for those women who answered to the King's Health Questionnaire. There was impact on all domains of quality of life.

\author{
Key words: Urinary \\ Incontinence. Urinary \\ Incontinence, Stress. \\ Nocturia. Quality of Life. \\ Health of the Elderly.
}

\section{INTRODUÇÃO}

De acordo com o censo realizado pelo IBGE em 2010, as mulheres constituem 51\% da população brasileira, sendo que $11,7 \%$ têm 60 anos de idade ou mais, o que corresponde a cerca de 11 milhões de pessoas. ${ }^{1}$

Estudos populacionais têm demonstrado que a prevalência de disfunções do assoalho pélvico (DAP), que incluem a incontinência urinária (IU), a incontinência anal (IA) e fecal e o prolapso genital (PG), aumenta de acordo com a idade e com as comorbidades apresentadas. Essas condições têm grande impacto negativo sobre a qualidade de vida (QV), afetando o contato social, a atividade laborativa, a higiene e a vida sexual das mulheres. ${ }^{2}$ Trazem também custos pessoais relacionados ao uso de absorventes, fraldas geriátricas e medicamentos, e oneram o sistema de saúde, por meio da demanda de consultas e internações para tratamento cirúrgico. ${ }^{3}$
A IU é uma DAP definida como o sintoma de qualquer perda involuntária de urina. Pode ser classificada clinicamente em três tipos principais: incontinência urinária de esforço (IUE), bexiga hiperativa $(\mathrm{BH})$ e incontinência mista (IUM). ${ }^{4}$

A IUE é o sintoma de perda de urina que acontece aos esforços (como tossir e espirrar) ou durante atividade física. A BH é uma síndrome clínica caracterizada pelo sintoma de urgência (necessidade súbita e imperiosa de urinar e difícil de conter), frequentemente associada à frequência (número excessivo de micções durante o dia, maior que o apresentado habitualmente) ou noctúria, podendo estar associada ou não a urge-incontinência (perda de urina associada à urgência). A incontinência mista, por sua vez, é a associação da perda aos esforços e da urgeincontinência. ${ }^{4} \mathrm{O}$ conhecimento e a classificação de tais disfunções são de fundamentalimportância para que se uniformize a linguagem na literatura, assim como o tratamento empregado. ${ }^{5}$ 
A prevalência de IU e seus três subtipos varia com a idade das mulheres e com o tipo de estudo realizado.6 Inquérito populacional realizado pela OPAS/OMS entre idosos com mais de 60 anos de idade na cidade de São Paulo encontrou prevalência de $26,2 \%$ de IU autorreferida entre as mulheres.7 No que se refere à IUE, estudos realizados em populações femininas brasileiras de diversas faixas etárias encontraram prevalências variando entre 12,7 e $35 \% .{ }^{8-10}$

Frequentemente associada a outros sintomas urinários, a queixa de noctúria é definida como interrupção do sono pelo desejo urinário pelo menos uma vez à noite, obrigando o indivíduo a levantar-se para urinar. ${ }^{11} \mathrm{O}$ sintoma tem sido negligenciado, mas possui efeitos deletérios específicos sobre o sono e, portanto, sobre a qualidade de vida (QV), e a resolução dos outros sintomas urinários não traz melhora concomitante da noctúria. ${ }^{12}$

Questionários têm sido desenvolvidos tanto para triagem de sintomas como para quantificar o impacto das DAPs sobre a QV. Dentre eles, podemos citar o International Consultation on Incontinence Questionnaire - Short Form (ICIQ-SF), questionário de triagem para IU traduzido e validado para o português em 2004.13 O King's Health Questionnaire (KHQ) é mais extenso e busca não só identificar os diferentes sintomas urinários como também seu impacto sobre a QV, e foi traduzido e validado para o português em $2003 .{ }^{14}$

Tendo em vista que a população feminina do município de Niterói-RJ constitui 53,6\% do total de habitantes, ${ }^{1}$ e que na população com mais de 60 anos a proporção de mulheres alcança $61 \%,{ }^{1}$ é fundamental o reconhecimento das DAPs por parte dos profissionais de saúde, para que sejam implementadas estratégias de saúde pública visando a sua prevenção e tratamento.

Este estudo teve como objetivos estimar a prevalência de IU e de seus subtipos (IUE, BH e IUM) na população feminina com mais de 60 anos de idade atendida na UBS da Engenhoca, Niterói-RJ, estimar a prevalência do sintoma de noctúria e avaliar o comprometimento da qualidade de vida causado por essas disfunções, usando como instrumentos o ICIQ-SF e o KHQ.

\section{MÉTODOS}

Trata-se de estudo observacional descritivo realizado em amostra de conveniência constituída pela população feminina que compareceu à Unidade Básica de Saúde (UBS) da Engenhoca, Niterói-RJ, no dia D da Campanha de Vacinação do Idoso contra o vírus influenza, realizada em 08 de maio de 2010. Foram incluídas no estudo mulheres com idade igual ou superior a 60 anos, sendo obedecidos os seguintes critérios de exclusão, identificados clinicamente no momento da abordagem da paciente e/ou por informações fornecidas por seu acompanhante: impossibilidade de ouvir e/ou entender as perguntas dos questionários devido a déficit cognitivo por doença mental, demências, síndromes genéticas ou congênitas, além de analfabetismo associado a surdez.

Estavam presentes para a campanha de vacinação 93 mulheres, das quais 71 concordaram em participar da pesquisa. Dessas, cinco tinham idades entre 55 e 59 anos e não foram incluídas, sendo o estudo desenvolvido com 66 participantes.

Para a triagem de IU, foi utilizado o ICIQ$\mathrm{SF}$, questionário que contém duas perguntas que investigam a frequência e a quantidade de perda urinária. Em seguida, o questionário se propõe a quantificar o impacto sobre a $\mathrm{QV}$ em escala analógica que varia de 0 (nenhum impacto sobre a QV) a 10 (grande impacto). Por último, são investigadas as situações em que ocorre perda urinária como, por exemplo, durante a tosse ou espirro, embora não questione sobre noctúria.

A versão para o português do ICIQSF foi validada em 2004 para aplicação em brasileiros de ambos os sexos e, devido a sua simplicidade e brevidade, constitui instrumento prático para utilização em contexto clínico ou epidemiológico. ${ }^{13}$ 
As mulheres que responderam positivamente a alguma pergunta do ICIQ-SF foram convidadas a responder o KHQ. Esse questionário contém 21 perguntas, que permitem calcular um escore de impacto da incontinência sobre a QV em nove domínios: percepção geral de saúde, impacto da incontinência, limitações das atividades diárias, físicas, sociais, relações pessoais, emoções, sono e disposição e medidas de gravidade. Além disso, identifica por meio de uma escala independente a presença e a intensidade dos sintomas urinários, como IUE, urgência, frequência e noctúria.

A cada resposta é atribuído um valor numérico entre 1 e 4 , proporcional à presença e à intensidade da queixa, que permite o cálculo de um escore que varia de 0 a 100 para cada um dos domínios, exceto para a percepção geral de saúde (valor das respostas entre 1 e 5). Quanto maior a pontuação, maior é o impacto sobre a qualidade de vida.

O KHQ foi validado para o português e adaptado para mulheres brasileiras em 2003. ${ }^{14}$ Apesar de ser um pouco mais extenso, também constitui instrumento prático para avaliação do impacto da IU na QV em ensaios clínicos ou epidemiológicos. Os questionários foram preenchidos pela própria participante ou, no caso de impedimento por deficiência visual, foi lido por um dos membros do grupo de pesquisa.
Foi utilizado para análise dos dados o programa Epi Info versão 3.5.2. As variáveis foram estudadas de maneira descritiva, por meio do cálculo de frequências absolutas (número de casos) e relativas (porcentagens) e, no caso da variável "idade", por meio do cálculo da média e desvio-padrão, mediana e valores mínimo e máximo. Para os escores de impacto da incontinência dos domínios do KHQ, foram calculados a média e o desvio-padrão.

O estudo foi aprovado pelo Comitê de Ética do Hospital Universitário Antônio Pedro, da Universidade Federal Fluminense (parecer $n^{\circ}$ 039/10), e as mulheres que aceitaram participar da pesquisa assinaram o Termo de Consentimento Livre e Esclarecido.

\section{RESULTADOS}

Participaram do estudo 66 mulheres, com

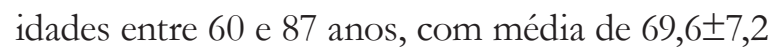
anos e mediana de 69 anos. Com o uso do ICQISF, foram identificadas 28 mulheres $(42,4 \%)$ com perda de urina. A idade dessas mulheres com IU variou entre 61 e 87 anos, com média de 70,7士8,69 anos e mediana de 69,5 anos. Referiam que a perda interferia na sua vida diária 12 idosas $(42,9 \%)$.

A distribuição da frequência e da quantidade de perda encontra-se nas tabelas 1 e 2 .

Tabela 1. Frequência da perda urinária em idosas com sintomas urinários, de acordo com o questionário ICIQ-SF. Niterói-RJ, 2010.

\begin{tabular}{lcc}
\hline \multicolumn{1}{c}{ Frequência } & $\mathrm{n}$ & $\%$ \\
\hline Nunca & 8 & 28,5 \\
Uma vez por semana ou menos & 8 & 28,5 \\
Duas a três vezes por semana & 2 & 7,2 \\
Uma vez ao dia & 0 & 0,0 \\
Diversas vezes ao dia & 5 & 17,9 \\
O tempo todo & 5 & 17,9 \\
Total & 28 & 100,0 \\
\hline
\end{tabular}


Tabela 2. Quantidade da perda urinária em idosas com sintomas urinários, de acordo com o questionário ICIQ-SF. Niterói-RJ, 2010.

\begin{tabular}{lcc}
\hline \multicolumn{1}{c}{ Quantidade de perda } & $\mathrm{n}$ & $\%$ \\
\hline Nenhuma & 5 & 17,9 \\
Uma pequena quantidade & 16 & 57,1 \\
Uma moderada quantidade & 6 & 21,4 \\
Uma grande quantidade & 1 & 3,6 \\
Total & 28 & 100,0 \\
\hline
\end{tabular}

As frequências dos subtipos de incontinência urinária (IUE, BH e IUM), de acordo com o ICIQ-SF, encontram-se na tabela 3.

Das 28 mulheres que referiram IU no questionário de triagem, 20 aceitaram responder ao KHQ. Com base nos sintomas referidos nesse questionário, foi feita nova classificação do subtipo de incontinência, cuja frequência não coincidiu com aquela estabelecida no questionário de triagem. O subtipo mais comum foi a IUM, presente em 11 mulheres (55\%), seguida de $\mathrm{BH}$, presente em oito mulheres $(25 \%)$ e IUE, que acometia três delas $(15 \%)$.

Avaliado apenas pelo KHQ, o sintoma de noctúria estava presente isoladamente em um caso (5\%), e era concomitante a outros sintomas urinários em 15 (tabela 4). Desse total de 16 mulheres, seis (37,5\%) apresentavam comprometimento no domínio sono e disposição do KHQ.

Tabela 3. Frequência dos subtipos de incontinência urinária identificados pelo questionário ICIQ-SF em idosas. Niterói-RJ, 2010.

\begin{tabular}{lcc}
\hline \multicolumn{1}{c}{ Diagnóstico clínico } & $\mathrm{n}$ & $\%$ \\
\hline IUE & 10 & 15,2 \\
BH & 8 & 12,1 \\
IUM & 7 & 10,6 \\
Perda dormindo & 1 & 1,5 \\
Perda o tempo todo & 1 & 1,5 \\
Sem resposta & 1 & 1,5 \\
Mulheres sem sintomas urinários & 38 & 57,6 \\
Total & 66 & 100,0 \\
\hline
\end{tabular}

Tabela 4. Frequência do sintoma de noctúria, de acordo com o questionário KHQ. Niterói-RJ, 2010.

\begin{tabular}{lcc}
\hline \multicolumn{1}{c}{ Noctúria } & $\mathrm{n}$ & $\%$ \\
\hline Ausente & 4 & 20 \\
Presente isoladamente & 1 & 5 \\
Noctúria + IUE & 1 & 5 \\
Noctúria + urgência/urge-incontinência & 14 & 70 \\
Total de casos & 20 & 100 \\
\hline
\end{tabular}


Foi observado impacto negativo dos sintomas de IU sobre a QV em todos os domínios do KHQ (tabela 5).
O domínio "relações pessoais" foi avaliado nas oito participantes que referiam ter atividade sexual $(40 \%)$. Duas apresentavam perda urinária durante o coito e uma delas referiu que a IU impactava negativamente o relacionamento com o parceiro.

Tabela 5. Impacto da incontinência urinária sobre a qualidade de vida de 20 mulheres idosas, de acordo com o questionário KHQ. Niterói-RJ, 2010.

\begin{tabular}{lcc}
\hline \multicolumn{1}{c}{ Domínio de QV } & Média \pm dp do escore de & $\mathrm{n}(\%)^{*}$ \\
\hline Percepção geral de saúde & $52,9 \pm 21,4$ & $17(85)$ \\
Impacto da incontinência & $50 \pm 22,5$ & $12(60)$ \\
Limitações das atividades diárias & $33,3 \pm 12,6$ & $8(40)$ \\
Limitações físicas & $35,7 \pm 20,2$ & $7(35)$ \\
Limitações sociais & $12,7 \pm 4,2$ & $7(35)$ \\
Emoções & $34,9 \pm 26,8$ & $7(35)$ \\
Sono e disposição & $54,7 \pm 32,9$ & $7(35)$ \\
Medidas de gravidade & $30,7 \pm 18,7$ & $18(90)$ \\
\hline
\end{tabular}

*Cada idosa pode ter impacto da IU em mais de um domínio.

\section{DISCUSSÃO}

Há disparidade entre os estudos que buscam avaliar a prevalência de IU entre mulheres, e um dos fatores que contribuem para isso é o método de recrutamento. ${ }^{5-11,15,16}$ Neste estudo, foi utilizada uma amostra de conveniência composta por 66 idosas que compareceram para vacinação contra influenza. A prevalência de IU, de acordo com as respostas ao questionário de triagem ICIQ-SF, foi mais próxima àquela observada em estudo com 40 mulheres recrutadas em instituições para idosos, 6 e mais elevada que a prevalência observada nos estudos feitos em populações brasileiras com idades semelhantes. ${ }^{7}$ Tal fato pode ser justificado pelas diferentes formas de recrutamento.

Dentre as 28 mulheres com IU, segundo o ICIQ-SF, o subtipo mais comum foi IUE. A literatura mostra que, quando a classificação da IU é feita com base nos sintomas, a IUE é realmente mais comum em mulheres adultas, independentemmete da idade. ${ }^{15} \mathrm{Com}$ a aplicação do KHQ, que investiga mais detalhadamente os sintomas urinários, o diagnóstico clínico mais comum, porém, foi incontinência mista. Nas faixas etárias mais avançadas, como é o grupo estudado, a prevalência de IUM e a $\mathrm{BH}$ cresce, o que pode justificar tal achado..$^{15}$ Outro fator a ser considerado é que o diagnóstico do subtipo de IU com base nos sintomas tem limitações. A principal delas é que a mulher incontinente pode não identificar suas queixas na linguagem médica utilizada pelo questionário, ainda que o mesmo tenha sido validado para a população em que está sendo utilizado, sugerindo que os questionários podem não ter sido entendidos de modo adequado. ${ }^{16}$

Independentemente do diagnóstico recebido, todas as mulheres apresentaram comprometimento da qualidade de vida pela perda urinária em pelo menos um dos domínios do KHQ. O domínio "medidas de gravidade" foi o mais afetado ( $90 \%$ dos casos), demonstrando o quanto a IU tem consequências 
psicológicas e altera o estilo de vida, já que a mulher incontinente se sente envergonhada, usa proteção ou até mesmo diminui a ingesta de líquidos para tentar minorar a perda urinária e suas consequências, como ter as roupas molhadas e exalar odor de urina. ${ }^{17}$

O domínio "percepção geral de saúde" esteve comprometido em 85\% dos casos. Idosas institucionalizadas com IU também apresentam comprometimento significativo desse domínio. Tais resultados refletem o prejuízo que a IU traz à percepção que a mulher tem de sua saúde em geral, esteja ela no seu ambiente familiar e social ou internada numa instituição para idosos. ${ }^{6}$

O domínio "impacto da incontinência" mostrou que, apesar de apresentarem perda urinária, $40 \%$ das mulheres não consideravam que isso afetava sua vida, o que provavelmente reflete o desenvolvimento de atitudes e comportamentos para o controle da continência e sua aceitação. ${ }^{18}$

O domínio "relações pessoais" foi avaliado em oito mulheres, uma vez que 12 dentre 20 participantes da pesquisa $(60 \%)$ que responderam ao KHQ negaram atividade sexual. A atividade sexual declina em idosos devido a vários fatores físicos, psicológicos e culturais, ${ }^{19}$ e as mulheres idosas tendem a dar menor importância ao sexo quando comparadas aos homens. ${ }^{20}$ A IU pode ser causa de constrangimento quando ocorre associada ao coito, e tal sintoma foi relatado por duas mulheres. No grupo em estudo, porém, a IU não parece ter sido um fator preponderante para diminuição da atividade sexual, já que as entrevistadas referiram que o motivo para isso não era a perda urinária.

Embora a avaliação do estado mental não mostre associação entre incontinência urinária e baixa autoestima, ${ }^{21} 35 \%$ das participantes apresentavam impacto da perda urinária no domínio "emoções", que avalia sintomas de depressão, ansiedade e autoestima.

O sintoma de noctúria esteve presente em $80 \%$ das mulheres que responderam ao KHQ, e em 93,8\% delas estava acompanhado de outros sintomas. A investigação de noctúria reveste-se de grande importância para a saúde do idoso. ${ }^{12}$ Embora seja muitas vezes negligenciada e considerada problema urológico ou ginecológico, tem inúmeras causas. Está associada a doenças crônicas, como hipertensão, diabetes, apneia do sono, doença renal crônica e ao uso de medicações para seu tratamento, o que habitualmente ocorre em populações idosas. ${ }^{22}$ Relaciona-se também a quedas ${ }^{23}$ e a maior mortalidade, quando comparada com indivíduos que não a apresentam. ${ }^{24}$

$\mathrm{O}$ acometimento do domínio "sono e disposição" em 35\% dos casos, com a média de escores mais elevada dentre os domínios do KHQ, pode ser efeito do sintoma de noctúria, já o que desejo urinário desperta a mulher durante a noite.

Ainda que os instrumentos utilizados no estudo tenham sido traduzidos para o português no Brasil e tenham sido validados no país, é possível que não tenham sido prontamente entendidos pelas mulheres que participaram do estudo, já que os resultados revelam informações contraditórias. Respondendo ao questionário ICIQ-SF, referiram que ocorria perda de urina em alguma situação (antes de chegar ao banheiro, durante esforços físicos, durante o sono ou sem razão óbvia) 28 mulheres; quando solicitadas a responder sobre a frequência da perda, porém, $28,5 \%$ delas referiram nunca perder urina, e cinco $(17,9 \%)$ referiram nenhuma quantidade de perda. Pode ser que a perda, nessas mulheres, tivesse frequência menor do que uma vez por mês e fosse em pequena quantidade. Como as perguntas estavam relacionadas às ultimas quatro semanas, a aparente incoerência pode refletir a limitação do instrumento utilizado para triagem de IU.

O estudo foi feito em uma amostra de conveniência, e é essa sua principal limitação. Além disso, a análise dos resultados sugere que os instrumentos utilizados não dispõem de linguagem adequada para que sejam entendidos com clareza e, portanto, que devem ser reavaliados. Apesar dessas limitações, a 
prevalência de IU sugere que atenção especial deva ser dispensada para a investigação de sintomas urinários em mulheres idosas, já que podem não só estar relacionados ao prejuízo da qualidade de vida, como também implicados em aumento de morbidade e mortalidade.

\section{CONCLUSÃO}

O estudo alcançou o objetivo de estimar a prevalência de incontinência urinária na população-alvo, enquantoa frequência de noctúria pôde ser estimada somente para as mulheres que aceitaram responder à versão brasileira do King's Health Questionnaire. Com a utilização da versão para o português do questionário de triagem International Consultation on Incontinence Questionnaire - Short Form, a incontinência urinária de esforço foi o tipo mais comum. Já a utilização do King's Health Questionnaire, mais detalhado, mostrou prevalência mais elevada de incontinência mista. Houve comprometimento da qualidade de vida em todos os domínios avaliados.

\section{REFERÊNCIAS}

1. Instituto Brasileiro de Geografia e Estatística. Sinopse do censo demográfico 2010 [Internet]. Rio de Janeiro: IBGE; 2011 [Acesso em 11 mai 2013]. 261 p. Disponível em http://www.ibge.gov.br/home/ estatistica/populacao/censo2010/sinopse.pdf

2. Bartoli S, Aguzzi L, Tarricone R. Impact on quality of life of urinary incontinence and overactive bladder: a systematic literature review Urology 2010;75(3):491-500.

3. Kannan H, Radican L, Turpin RS, Bolge SC. Burden of illness associated with lower urinary tract symptoms including overactive bladder/urinary incontinence. Urology 2009;74(1):34-8.

4. Haylen BT, de Ridder D, Freeman RM, Swift $\mathrm{SE}$, Berghmans B, Lee J, et al. An International Urogynecological Association (IUGA)/International Continence Society (ICS) joint report on the terminology for female pelvic floor dysfunction. Neurolol Urodyn 2010;29(1):4-20.

5. Amaro JL, Macharelli CA, Yamamoto H, Kawano PR, Padovani CV, Agostinho AD. Prevalence and risk factors for urinary and fecal incontinence in Brazilian women. Int Braz J Urol 2009;35(5):592-7.

6. Pitangui ACR, Silva RG, Araújo RC. Prevalência e impacto da incontinência urinária na qualidade de vida de idosas institucionalizadas. Rev Bras Geriatr Gerontol 2012;15(4):619-26.

7. Tamanini JTN, Lebrão ML, Duarte YAO, Santos JLF, Laurenti R. Analysis of the prevalence of and factors associated with urinary incontinence among elderly people in the Municipality of São Paulo, Brazil: SABE Study (Health, Wellbeing and Aging). Cad Saúde Pública 2009;25(8):1756-62.

8. Mendonça M, Reis RV, Macedo CBMS, Barbosa KSR. Prevalência da queixa de incontinência urinária de esforço em pacientes atendidas no serviço de ginecologia do Hospital Júlia Kubitschek. J Bras Ginecol 1997;107(5):153-5.

9. Guarisi T, Pinto-Neto AM, Osis MJ, Pedro AO, Costa-Paiva LHS, Faúndes A. Procura de serviço médico por mulheres com incontinência urinária. Rev Bras Ginecol Obstet 2001;23(7):439-43.

10. Brito LGO, Brito LMO, Chein MBC, Malheiros ESA, Duarte TB, Pinto-Neto AM. Stress urinary incontinence in climacteric women in a northeastern Brazilian municipality: a household survey. Int Urogynecol J 2012;23(5):639-45.

11. Burgio KL, Johnson TM 2., Goode PS, Markland AD, Richter HE, Roth DL, et al. Prevalence and correlates of nocturia in community-dwelling older adults. J Am Geriatr Soc 2010;58(5):861-6.

12. Van Kerrebroeck PEV, Dmochowski R, Fitzgerald MP, Hashim H, Norgaard JP, Robinson D, et al. Nocturia research: current status and future perspectives. Neurourol Urodyn 2010;29(4):623-8.

13. Tamanini JTN, Dambros M, D'Ancona CAL, Palma PCR, Netto Jr NR. Validação para o português do "International Consultation on Incontinence Questionnaire-Short Form” (ICIQ-SF). Rev Saúde Pública 2004;38(3):438-44.

14. Tamanini JTN, D'Ancona CAL, Botega NJ, Netto Jr NR. Validação do "King's Health Questionnaire" para o português em mulheres com incontinência urinária. Rev Saúde Pública 2003;37(2):203-11. 
15. Hunskaar S, Burgio K, Diokno A, Regula Herzog A, Hjalmas K, Lapitan MC. Epidemiology and natural history of urinary incontinence in women. Urology 2003;62(4 Suppl 1):16-23.

16. Palva K, Nilsson CG. Prevalence of urinary urgency symptoms decreases by mid-urethral sling procedures for treatment of stress incontinence. Int Urogynecol J 2011;22(10):1241-7.

17. Minassian V, Stewart W, Hirsch A, Kolodner K, Fitzgerald M, Burgio K, et al. The role of urgency, frequency, and nocturia in defining overactive bladder adaptive behavior. Neurourol Urodyn 2011;30(3):406-11.

18. Higa R, Lopes MHBM, Turato ER. Significados psicoculturais da incontinência urinária feminina: uma revisão. Rev Latinoam Enferm 2008;16(4):779-86.

19. Gradim CVC, Sousa AMM, Lobo JM. A prática sexual e o envelhecimento. Cogitare Enferm 2007;12(2):204-13.
20. Bastos CC, Closs VE, Pereira AMVB, Batista C, Idalêncio FA, de Carli GA, et al. Importância atribuída ao sexo por idosos do município de Porto Alegre e associação com autopercepção de saúde e sentimento de felicidade. Rev Bras Geriatr Gerontol 2012;15(1):87-95.

21. Melo BES, Freitas BCR, Oliveira VRC, Menezes RL. Correlação entre sinais e sintomas de incontinência urinária e autoestima em idosas. Rev Bras Geriatr Gerontol 2012;15(1):41-50.

22. Gulur DM, Mevcha AM, Drake MJ. Nocturia as a manifestation of systemic disease. BJU Int 2011;107(5):702-13.

23. Vaughan CP, Brown CJ, Goode PS, Burgio KL, Allman RM, Johnson TM 2. The association of nocturia with incident falls in an elderly communitydwelling cohort. Int J Clin Pract 2010;64(5):577-83.

24. Kupelian V, Fitzgerald MP, Kaplan SA, Norgaard JP, Chiu GR, Rosen RC. Association of nocturia and mortality: results from the Third National Health and Nutrition Examination Survey. J Urol 2011;185(2):571-7. 